\title{
Das ökonomische Dilemma
}

\author{
Friedrich Overkamp Anja Bröker \\ Oncologianova GmbH, Recklinghausen, Deutschland
}

Schlüsselwörter

Unterfinanzierung · Klinische Studien · Finanzierung • Studien-GmbH $\cdot$ Vergütung $\cdot$ Kosten

\section{Zusammenfassung}

Die Bereitschaft von Praxen und Kliniken für ein Engagement im Bereich der Klinischen Forschung ist in den letzten 10 Jahren deutlich gestiegen. Es macht sich jedoch zunehmend Ernüchterung breit im Hinblick auf die eklatante Unterfinanzierung klinischer Studien. Der vorliegende Beitrag möchte aufgrund praktischer Erfahrungen Denkanstöße und Impulse für eine Neuorientierung der Vergütung setzen. Ursachen und Hintergründe der Unterfinanzierung werden erläutert, insbesondere werden die anfallenden Kosten eingehend dargelegt. Die Etablierung von Werkzeugen zur Kostenkalkulation erscheint dringend notwendig, erste Ansätze dazu gibt es bereits.

\section{Das Dilemma}

Die Beteiligung an der klinischen Forschung ist heute essenzieller Bestandteil einer hochwertigen onkologischen Einrichtung. Onkologische Schwerpunktpraxen haben in der letzten Dekade vielfach Tochtergesellschaften zur Durchführung klinischer Studien gegründet, meist in der Rechtsform einer Gesellschaft mit beschränkter Haftung $(\mathrm{GmbH})$. Über diese «Studien-GmbHs» bieten sie den Patientinnen und Patienten die Teilnahme an Studien aller 4 Prüfphasen an, insbesondere an Phase-II- und -III-Studien.

Die Motivation ist hoch, die meisten Einrichtungen sind sehr professionell aufgestellt, die Rekrutierung läuft bei vielen Studien bemerkenswert gut und die ideellen und fachlichwissenschaftlichen Vorteile sind für alle Beteiligten unüber-

\author{
Keywords \\ Underfinancing · Clinical trials · Financing · \\ Study company with limited liability - Reimbursement . \\ Costs
}

\section{Summary}

\section{The Economic Dilemma}

Clinical studies have been situated very well in Germany during the last 10 years; oncologists in practices and clinics are highly motivated. However, the financing of clinical trials is desolate so that disillusionment spreads in many centers. This paper would like to give some impetus to the discussion on better and more adequate financing. Facts and backgrounds concerning unsatisfactory financing of clinical trials are demonstrated; costs are analyzed in a detailed way. It seems to be very important to establish tools for a better calculation of costs. First approaches have been defined. sehbar. Bei aller Motivation und trotz des hohen Engagements macht sich jedoch zunehmend Ernüchterung breit im Hinblick auf die eklatante Unterfinanzierung der meisten Studien.

Vielfach ist die Honorierung nicht einmal andeutungsweise kostendeckend! Dies ist alarmierend, da nicht nur die Einrichtungen ökonomisch in Gefahr sind, sondern mit ihnen vor allem die Zukunft des Forschungsstandorts Deutschland.

In den Kliniken bestehen das Problem der Unterfinanzierung und die drohenden Konsequenzen in gleicher Weise. Das ökonomische Dilemma dort betrifft insbesondere Therapieoptimierungsstudien bei akuter lymphatischer Leukämie (ALL), akuter myeloischer Leukämie (AML), chronischer myeloischer Leukämie (CML), Morbus Hodgkin usw., für die faktisch eine ethische Verpflichtung besteht teilzunehmen,

\section{KARGER \\ Fax +497614520714 \\ Information@Karger.com}

www.karger.com (c) 2013 S. Karger GmbH, Freiburg

0378-584X/13/3614-0041\$38.00/0

Accessible online at:

www.karger.com/onk
Dr. med. Friedrich Overkamp

Oncologianova $\mathrm{GmbH}$

Gesellschaft für Innovationen in der Onkologie

Springstraße 24, 45657 Recklinghausen, Deutschland

www.oncologianova.de 
aber praktisch keine Vergütung (50-70 EUR pro Patient) erfolgt.

Im Folgenden sollen aufgrund der Erfahrungen einer praxisassoziierten Studien-GmbH Denkanstöße und Impulse für eine Neuorientierung der Vergütung gesetzt werden. Die Ansätze sind jedoch prinzipiell auch auf Kliniken und ihre Studieneinrichtungen zu übertragen.

\section{Die Diskrepanzen}

Grundsätzlich sind sowohl bei Investigator-initiierten Studien (IIT) als auch bei Pharmaindustrie-gesponserten Studien Probleme der Unterfinanzierung zu verzeichnen, bei IIT jedoch in weitaus größerem Umfang, bei Industriestudien seltener und nicht ganz so gravierend.

Für Phase-II/III-Studien liegt die Vergütung zwischen etwa 10000 und 30000 EUR, die Spanne ist somit sehr groß. IIT werden mit durchschnittlich etwa 750 EUR vergütet, oft noch deutlich weniger.

Da nichtinterventionelle Studien (NIS) und Registeruntersuchungen bei deutlich überschaubareren Anforderungen und geringerem Dokumentationsaufwand wesentlich besser honoriert werden, müssen sie oft zur Gegenfinanzierung mangelhaft honorierter IIT herangezogen werden. Die Unterdeckung ist bei einigen Studien jedoch inzwischen so bedeutsam, dass sie mit Honoraren aus NIS und Registern nicht mehr kompensiert werden kann.

\section{Die Ursachen}

\section{Externe Kosten}

Ein Hauptproblem besteht darin, dass neben der protokollgerechten Patientenbehandlung und Dokumentation durch die «hauseigenen» Prüfärzte (bisher: Hauptprüfer/Principal Investigator (PI) und Subinvestigator (SI), neuerdings: «Prüfgruppe») und Study Nurses (SN) diverse externe Kosten anfallen: Dies sind insbesondere Kosten für Labor, Kardiologie, Pathologie, Radiologie und zubereitende Apotheke bzw. Herstellerbetrieb.

Labormediziner, Kardiologen, Radiologen und Pathologen berechnen ihre studienbezogenen Leistungen gegenüber der Studien-GmbH in der Regel nach der Gebührenordnung für Ärzte (GOÄ), Pathologen fordern zusätzlich eine Pauschale für die Zubereitung und den Versand von Gewebeschnitten oder Tumorblöcken, Apotheker berechnen die vorgeschriebene Taxe für die Zubereitung der Studientherapie.

Diese Kosten übersteigen das Gesamt-Fallhonorar einer IIT oft um ein Vielfaches. Zu fordern sind daher entweder separate Regelungen und entsprechende Verträge für die genannten externen Leistungserbringer oder eine entsprechende Erhöhung der Gesamtvergütung. Bei industriege- sponserten Studien sind separate Verträge mit Apotheke oder radiologischer Einrichtung teilweise schon umgesetzt worden, bei IIT wurde dies bisher stets abgelehnt.

Ein logisches und adäquates Vorgehen wäre es, diejenigen Untersuchungen, die als Standard angesehen werden, nicht aus den Studien-Endgeldern zu bezahlen, sondern den Krankenkassen in Rechnung zu stellen. Hierzu zählen unter anderem Staging-Untersuchungen alle 2-3 Monate, die RoutineLaboruntersuchungen oder auch Echokardiogramme. In der Regel wird bei IIT eine Standardtherapie in Kombination mit einer Prüfsubstanz oder Placebo verabreicht, sodass die Kosten für die Standardtherapie inklusive Begleituntersuchungen genauso über die Kassen abgerechnet werden sollten, wie es im Falle einer Behandlung außerhalb der Studie auch der Fall wäre. Dieses Prozedere wird zwar schon häufig praktiziert, rechtsverbindlich ist es derzeit aber noch nicht.

In diesem Zusammenhang ist festzuhalten, dass nicht zuletzt die Krankenkassen erhebliche Vorteile von der Durchführung von Studien haben. Diese bestehen nicht nur in einer besonders qualitätsgesicherten Betreuung der Patienten, sondern auch darin, dass Kosten und Nutzen einer innovativen Therapie kontrolliert überprüft werden und es deshalb auch gelingen kann, möglicherweise kostentreibende, aber nicht sehr wirksame Medikamente von der Zulassung auszuschließen.

\section{Interne Kosten - zentrumsspezifisch}

Die internen Kosten müssen getrennt nach zentrumsspezifischen und patientenspezifischen Kriterien bewertet werden. In die Zentrumshonorierung müssen Anteile für Personalkosten, Energiekosten, Raummiete, Büromaterial, EDV, Internet, E-Mail, Telefon, Fax usw. eingerechnet werden.

Aufgrund der immer höheren Anforderungen im Rahmen der Good Clinical Practice (GCP) sind z.B. adäquate Räumlichkeiten für die Studieneinrichtung vorzuhalten, abschließbar und ohne Zugang für Unberechtigte. Für die im Studienzentrum gelagerte Studienmedikation müssen ebenso geeignete Räume mit abschließbaren (Kühl-)Schränken, gegebenenfalls mit Temperaturkontrollen, bereitgestellt werden.

Zentrumsspezifische, interne Kosten werden auch durch das Monitoring ausgelöst. Für den Monitorbesuch sollte ein separater Raum mit EDV-Ausstattung zur Verfügung stehen, die zuständige SN muss entsprechende Zeit zur Besprechung einplanen und steht in dieser Zeit für Dokumentation und Betreuung der Studienpatienten nicht zur Verfügung.

Die in letzter Zeit in rasch zunehmendem Ausmaß zu beobachtende Umstellung des Monitorings auf häufigere telefonische Kontakte zwischen Monitor und $\mathrm{SN}$ bedeutet für das Studienzentrum den Entzug weiterer Ressourcen und die Bürde zusätzlicher Kosten, da sich die SN zu festen Telefonterminen bereithalten müssen.

In letzter Zeit ist auch ein Trend zur Durchführung von Initiierungsvisiten über Telefonkonferenzen, teilweise Webi- 
nar-basiert, zu beobachten. Dies ist für das Studienzentrum kaum noch zu bewerkstelligen, da PI und SN, eventuell auch SI, gleichzeitig am Telefon erwartet werden - ein mit der Patientenversorgung nicht mehr zu vereinbarender Zeit- und Ressourcenverbrauch.

Schließlich sei im Zusammenhang mit den zentrumsspezifischen Kosten auch erwähnt, dass diese Kosten natürlich auch dann anfallen, wenn keine Patienten in die Studie eingeschlossen werden.

\section{Interne Kosten - patientenspezifisch}

Die patientenspezifische Honorierung muss grundsätzlich die Kosten für die Dokumentation, Organisation, Aufklärung und Behandlung im Rahmen einer Studie abdecken. Hierzu haben wir im Folgenden einige spezielle Vorschläge zusammengestellt, die wir aufgrund unserer Erfahrungen als orientierende Richtgrößen verstanden wissen möchten.

Der Stundensatz für Ärzte und SN sollte sich dabei grundsätzlich an den allgemein gültigen Sätzen der GOÄ und der Krankenschwestertarife (Arbeitgeber-Brutto) orientieren. Im Hinblick auf die ärztlichen Leistungen ist allerdings zu diskutieren, ob der Routinesatz nach GOÄ im Einzelfall ausreicht. Denn es wird ja nicht nur korrektes medizinisches Handeln, sondern über das normale Maß hinausgehende Beratung, qualitätsgerechte Dokumentation und Adhärenz an das Studienprotokoll erwartet.

\section{Vor der Initiierung}

Vorbemerkung: Die Angabe pauschaler Zeiten für bestimmte Tätigkeiten ist schwierig, die Variabilität ist naturgemäß hoch. Dennoch sind im Folgenden Durchschnittswerte angegeben, die eine hinreichend genaue Diskussionsgrundlage darstellen sollen. Letztlich wird die Erfassung der für die verschiedenen Prozesse notwendigen Zeiten nur durch flexible Werkzeuge (Tools) möglich sein, in denen individuelle Studienmerkmale für jede einzelne Studie berücksichtigt werden können.

Kosten für Tätigkeiten vor der Initiierung (confidential agreement, feasibility check, pre-study visit usw.) sollten separat und zeitnah zu Beginn vergütet werden (start-up fee).

Die Lektüre des Protokolls und der Einverständniserklärung(en) nimmt bei den PI etwa 2 Stunden, bei den SI 1 Stunde und bei den SN 2-3 Stunden pro Studie in Anspruch, je nach Aufwand und Komplexität.

Die Bereitstellung der Dokumente für Sponsor und Ethikkommission, gegebenenfalls Bundesamt für Strahlenschutz, im Zusammenhang damit notwendige Telefonate, Fax- und E-Mail-Kommunikation, beschäftigt die SN im Vorfeld einer jeden Studie für etwa 4-5 Stunden, die Ärzte sind hier zu je 15-30 Minuten beteiligt für Unterschriften, Kontrollen, Bestätigungen usw.

Beim Pre-Study Visit ist der PI mit etwa 30 Minuten und die SN mit etwa 6 Stunden pro Studie beteiligt.
Die ökonomische Kalkulation im Vorfeld der Initiierung, z.B. die Erfassung der angebotenen Studie in Kostenerfassungstools, beschäftigt die geschäftsführende SN und/oder die geschäftsführenden Ärzte weiterhin für etwa 2-3 Stunden pro Projekt.

Ein wesentlicher Faktor in der Vorphase ist die Vorbesprechung der angebotenen oder geplanten Studie im Planungsboard der Studieneinrichtung, des Weiteren unmittelbar nach Initiierung die Vorstellung der Studie in den Tumorkonferenzen. Diese Tätigkeit obliegt den Prüfärzten und kann mit mindestens 2-3 Stunden PI-Tätigkeit pro Studie berechnet werden (Fahrzeiten zu den Sitzungen nicht eingerechnet).

\section{Initiierung}

Kosten für die Initiierung stellen ebenfalls einen einmaligen Aufwand dar.

Die Initiierungsvisite selbst nimmt den PI etwa 2-3 Stunden, die SI jeweils 1 Stunde und die SN jeweils 8 Stunden in Anspruch.

\section{Visiten}

Im Gegensatz zu den einmalig anfallenden Kosten vor und während der Initiierung ist bei den Studienvisiten der Aufwand pro Patient zu berechnen.

Screeningvisiten (mittelhoher Aufwand), Baseline Visit (sehr hoher Aufwand wegen der initialen Diagnostik und der Aufklärung), Visiten in der Interventionsphase (hoher bis sehr hoher Aufwand) sowie Visiten in der Follow-up-Phase (niedriger Aufwand) sind unterschiedlich zu bewerten. (Anmerkung: Gegebenenfalls muss auch noch zwischen stationärer und ambulanter Behandlung unterschieden werden.) Screening und Baseline sind besonders zeit- und arbeitsintensiv für die SN, Visiten in der Interventionsphase erfordern eine besondere Beanspruchung der Prüfärzte.

Anamneseerhebung, Dokumentation, Erfassung der Vitalzeichen, ärztliche Untersuchung, Blutabnahmen, Veranlassung der Restaging-Untersuchungen usw. müssen für jede Studie und für die beteiligten Berufsgruppen bewertet werden. Im Durchschnitt kann jedoch eine Gesamtbeanspruchung der SN von 3,5 Stunden und des PI von 1-2 Stunden in der Screeningphase zugrunde gelegt werden, in der Baseline sind es etwa 4,5 Stunden für die SN und 1 Stunde für den PI/ SI.

Für Visiten in der Interventionsphase sind für den Prüfarzt jeweils 0,5 Stunden, für die SN 1 Stunde anzusetzen (Vorbereitung, Blutentnahme, Anmeldung Untersuchungen, Vitalzeichen, Dokumentation, Meldung SAE (Serious Adverse Events, schwerwiegende unerwünschte Ereignisse), Fax, Telefonate, E-Mails, Archivierung usw.). In der Follow-upPhase reduziert sich der Aufwand in der Regel um etwa 50\%.

Beim Punkt «Blutentnahme» muss die Vorbereitung ebenso berücksichtigt werden wie die eigentliche Durchführung sowie gegebenenfalls die Aufbereitung der Proben und der Probenversand. 
Der Dokumentationsaufwand ist natürlich abhängig von der unterschiedlichen Komplexität der Anforderungen, von Morbidität und Komorbidität, der (Ko-)Medikation, den auftretenden unerwünschten Ereignissen (Adverse Events) usw. Eindeutig korreliert er mit dem Ausmaß der Morbidität des Studienpatienten. Die Zeitspanne für eine professionelle und korrekte Dokumentation inklusive SAE-Management kann pro Patient beträchtlich schwanken.

Ein weiterer nicht zu unterschätzender Punkt während der Therapiephase sind schließlich Fahrtkostenerstattungen, die von den Patienten häufig gefordert werden und naturgemäß im Rahmen von Studien nicht den Krankenkassen angelastet werden können. Die Fahrtkosten sollten zeitnah und nicht erst am Ende der Studie erstattet werden.

\section{Investigator-Meetings, Webmeetings, Case Report Form-} und Protokoll-Training

Die Teilnahme an Investigator-Meetings oder Web-basierten Konferenzen (Webmeetings) kann pro PI und SN mit jeweils 6-8 Stunden pro Studie veranschlagt werden (Fahrtkosten nicht eingerechnet).

Zunehmende Bedeutung gewinnt bei industriegesponserten Studien das Case Report Form(CRF)- und ProtokollTrainung, das von SN und Prüfärzten getrennt absolviert werden muss. Diese Tätigkeit wird ebenfalls unterschätzt und offenbar in den bisherigen Vergütungsmodellen nicht berücksichtigt. Etwa 1-2 Stunden pro Studie müssen diesbezüglich für PI und SI sowie etwa 5-6 Stunden für SN angesetzt werden.

\section{Queries, CIOMS, SUSAR}

Der laufende Aufwand für PI und SN während der gesamten Studie ist hoch und wird eklatant unterbewertet. Dies gilt insbesondere für die Sichtung und Bewertung der Suspected Unexpected Serious Adverse Reactions (SUSAR) laut Council for International Organizations of Medical Sciences (CIOMS), die Informationsweitergabe an die SI, wiederholte Bewerbung der Studie in Tumorkonferenzen usw. Hierfür sind durchschnittlich für den PI 2 Stunden/Monat und für die SN etwa 3 Stunden/Monat zu veranschlagen. SUSAR sind natürlich abhängig von der Patientenzahl in der Gesamtstudie, von der Entwicklungsphase des Studienmedikamentes und von der erwarteten Häufigkeit schwerwiegender Ereignisse.

Für die Beantwortung von Queries (Nachfragen) können im Schnitt etwa 30-40\% der Dokumentationszeit bei kommerziellen Studien und etwa $25 \%$ bei IIT veranschlagt werden.

Im Zusammenhang mit SAE/SUSAR sei noch darauf hingewiesen, dass von Pharmafirmen bzw. von deren Clinical Research Organizations (CRO) zum Zwecke der Absicherung alle SUSAR/SAE eines Medikamentes (auch aus verschiedenen Studien, an denen man gar nicht teilnimmt) unge- filtert an die Studienzentren weitergeleitet werden. Dies führt $\mathrm{zu}$ zentimeterdicken SUSAR-Stapeln und/oder Dutzenden Hunderten von E-Mails pro Woche, die alle gesichtet und unterzeichnet werden müssen.

\section{Audits/Inspektionen}

Für eventuell anfallende Audits oder Inspektionen sollte für jede Studie eine fakultative zusätzliche Vergütung vereinbart werden, da ein Zentrum, das an einem Audit teilnehmen muss, einen deutlich höheren Aufwand hat als ein Zentrum, das an der gleichen Studie teilnimmt, aber nicht auditiert wird.

\section{Close-out}

Der Close-out Visit beschäftigt den PI etwa 30 Minuten, die SN etwa 3-4 Stunden. Nachdokumentationsanforderungen zum Datenbankschluss, Archivierung usw. sollten bei den SN nochmals mit etwa 4-5 Stunden veranschlagt werden.

\section{Diskussionsvorschlag}

Bereits mit der Studiensynopse sollte dem Studienzentrum das vorgesehene Prüfhonorar mitgeteilt werden, damit es in die Machbarkeitsanalyse einfließen kann. Die ökonomische Betrachtung ist genauso von Bedeutung wie die Frage der Rekrutierungsprognose oder der Machbarkeit bestimmter Untersuchungen und Therapien.

Basis ökonomischer Berechnungen sollten sein: die Zahl der voraussichtlich eingebrachten Patienten, die Zahl der beteiligten Prüfärzte, die Zahl der beteiligten SN und das Ausmaß der Morbidität des Studienpatienten.

Kalkuliert man die nur für den jeweils einmaligen Aufwand in den Bereichen Pre-Study, Initiierung und Close-out anfallenden Kosten mit spitzer Feder, ergeben sich allein daraus bei jeder Studie Basiskosten von 3500-5000 EUR (unterschiedlich für IIT und gesponserte Studien). Rechnet man die oben erläuterten Kosten für die Visiten sowie die übrigen zentrumsspezifischen Kosten hinzu, kommt man zu dem Schluss, dass jede IIT unter 5500 EUR Gesamthonorar zwangsläufig eine Unterdeckung aufweisen muss.

Erste Werkzeuge zur Kostenschätzung sind bereits erarbeitet worden, z.B. STUDGET vom Zentrum für Klinische Studien der Universität Köln (studget.clinicalsite.org). Sie führen $\mathrm{zu}$ ähnlichen Bewertungen und können zur Kostenkalkulation herangezogen werden.

\section{Disclosure Statement}

Dr. Friedrich Overkamp und Anja Bröker haben keine Interessenkonflikte. 